\title{
Scaffolding the Development of an Inquiry-Based (Science) Classroom
}

\author{
Jennifer Holbrook \& Janet L. Kolodner \\ College of Computing, Georgia Institute of Technology, Atlanta, GA 30332 \\ Tel: 404-894-3285, Fax: 404-894-5041 \\ Email: \{holbrook, jlk\}@cc.gatech.edu
}

\begin{abstract}
Our experience with Learning by Design, an inquiry-based and design-based approach to middle-school science has uncovered several problems teachers have introducing students to an inquiry approach. Teachers find it hard to help students learn content at the same time they are learning skills and processes required for inquiry and design. As well, many students need time to adapt to a classroom situation in which they must take initiative. In response to this need, we've created a "launcher unit" for our LBD classrooms called Apollo 13. Students watch a movie where they see scientists and engineers engaging in collaboration, communication, informed decision making, inquiry, and design, and then they engage in a series of short activities that focus on learning these complex skills. The activities provide an anchor for the rest of the year's activities in science class. While designed for LBD, the approach may be appropriate for other inquiry approaches as well.
\end{abstract}

Keywords: science education, middle school, learning environments, cognitive science

\section{Setting the Context: The Learning-by-Design}

Learning By Design (Kolodner et al, 1998, Hmelo et al, 2000) is an NSF-funded effort to promote the development of inquiry-based science classrooms in contemporary school settings. Science learning is achieved through addressing a major design challenge (such as building a self-powered car that can go a certain distance over a certain terrain) (Kolodner et al, 1998). To address a challenge, class members develop designs, build prototypes, gather performance data and use other resources to provide justification for refining their designs, and they iteratively investigate, redesign, test, and analyze the results of their ideas. They articulate their understanding of science concepts, first in terms of the concrete artifact that they have designed, then in transfer to similar artifacts or situations, and finally to abstract principles of science. The design challenge provides them a reason to ask questions, provides focus for their investigations, and provides a context for applying what they are learning and analyzing how well they've learned it. To make all this work, we have developed curriculum materials, professional development for participating teachers, and a system of classroom rituals. LBD units are written for middle-school science classes, to be used for a portion of the school year. Our current units cover topics in physical science (Newton's laws of motion, simple machines, and work and energy) and earth science (rock formation and properties, erosion).

Over the last five years, we have supported the development and implementation of LBD units by almost 25 teachers. All implementations have taken place in public schools, and we have made sure that the implementations included a wide range of settings (urban, suburban and near-rural communities; schools with large, medium, and small racial minorities; student populations with high, medium, and low income; large and small school districts, classes of normal, at-risk, and gifted students; new and experienced teachers, and working both with teachers who have experience with inquiry-based teaching and those who do not and with teachers who have strong background in the subject matter of the curriculum and those who do not). We have used many methods to evaluate the success of the curriculum (see Fasse \& Kolodner, 2000), including frequent discussions with implementing teachers and their supervisors, ethnographic observations in the classrooms (Gertzman \& Kolodner, 1996, Hmelo et al., 2000), student surveys and interviews, analysis of the development of student's metacognitive skills and science thinking (Puntambekar \& Kolodner, 1998), assessment of student learning both through standardized test items in a pre/post-implementation design using matched comparison classes, and results from performance-based assessment tasks. These evaluations show that in LBD classes, students indeed learn the designated science as well as or better than their peers in traditional classes. Of more interest is that they also learn 
science process skills, collaboration, communication, and planning skills exceeding those of their peers (in preparation).

We have also discovered several interesting problems: (i) Teachers find it difficult to help students learn science concepts at the same time they are being introduced to the processes involved in designing, doing science, communicating, collaborating, and so forth. Teachers prefer that students have some minimal expertise with these complex processes before using them to learn science content. (ii) Students are not used to the kinds of collaboration, communication, and learner-centered skills that we want them to use in the classroom; they need time to get comfortable with being active learners. (iii) Teachers who aren't used to student-inquiry-centered teaching learn facilitation skills better with short activities that center on scientific process rather than content.

Based on advice from several of our pioneering teachers, we've developed a "launcher" unit called Apollo 13 to achieve solutions to all three of these problems. The three-to-four-week unit is intended to replace the usual introductory weeks of a traditional curriculum which typically focus on explication of the scientific method, the culture of science, measuring skills, and safety procedures. The launcher unit is designed to cover these introductory topics and at the same time create a culture of collaboration and inquiry and introduce LBD rituals. It allows both the teacher and the class to ease into this new way of doing things and provides, as well, a shared set of anchoring experiences that can be referred back to repeatedly during the year as students actively engage in science activities. We present an overview of the LBD classroom, the rationale for our "launcher" unit, and the unit we've designed. We suspect that this approach will prove useful not only for LBD but for introducing the wide range of problem-based and project-based curricula that are currently under development.

\section{Elements of the Learning-By-Design Classroom}

\section{Teacher and Student Roles}

In LBD classrooms, students work on a design challenge in small groups and share their knowledge with the rest of the class collaboratively. Groups rather than teachers develop expertise and disseminate their knowledge to classmates. During whole-class discussion, the class develops guidelines for experimentation, including classuniversal procedures, measurements, and so on. Student groups carry out investigations keeping those guidelines in mind and update them as they discover them to be inadequate. The teacher's role is less about setting and enforcing specific tasks and more about moderating discussions during which the class identifies what it needs to be doing. A teacher sets up time and resource guidelines, which may be modified with justification. The teacher's expertise in science methodology and science content is used as one of many resources, as is the textbook. While students must learn terminology, formulae, and methods, they do so in service of and during practice with their design goals, not simply as fodder for exams.

\section{Classroom Culture}

A culture is a body of customary beliefs, mutual goals, rituals, social forms, language and artifacts that unify and provide distinction for a group. LBD works best when (i) the culture values collaboration in learning and sharing knowledge; (ii) projects go through multiple iterations, supporting and valuing the articulation of lack of knowledge, misconceptions, and failures; (iii) a teacher's leadership involves scaffolding inquiry rather than automatically providing information, (iv) the culture values members' diverse talents, perspectives, and knowledge as assets to collaboration and learning, (v) students are encouraged to make, specify, and justify their decisions, and (vi) the teacher is confident about allowing the students to set out on their own paths. The classroom culture is so important to this mode of teaching that a major LBD teacher-development goal is to educate teachers in how to engender this culture in the classroom.

\section{Rituals that Support Inquiry-Based Learning}

LBD's system of activities is designed to support a cycle of designing and testing solutions to the challenge presented in the curriculum. These rituals include messing about with challenge materials and/or artifacts. Messing about helps students recognize and articulate what they already know about the challenge and helps them generate relevant questions and initial solutions. Whiteboarding (Barrows, 1986) is used with the whole class to develop issues for investigation and to keep track of what is learned throughout the challenge. Groups get feedback on their initial ideas from the teacher and other class members at pin-up sessions (Kolodner et al, 1998). The groups iteratively build and test their designs, gathering data on the performance of each prototype and using the data to revise their designs. Gallery walks (Kolodner et al, 1998) - presentations of in-progress designs and finished 
products -- allow groups to offer one another constructive feedback, to share expertise, and to garner inspiration. The class also develops better understanding of the underlying science through reasoning from cases (the current class' designs and existing designs in the world), and through developing rules of thumb as a class.

\section{Specific Classroom Needs}

Both teacher's comments and ethnographer's observations during early implementations of Learning By Design content curriculum helped us identify a set of related problems that seemed to be impediments to learning in a highly-collaborative, student-centered, and hands-on classroom:

1) Groups too often did not work well together.

2) An artifact might be successfully completed by a group without the individuals all understanding the rationale for its design, the method of its construction, or how it embodied the science.

3) Students needed a great deal of help with the scientific method and with understanding the advantages and disadvantages of models. Yet teachers were not experienced at developing these skills "on-line".

4) Students ignored or didn't understand what it meant to meet a challenge.

5) Competition between groups kept the students from acknowledging design flaws and from discussing and discovering the design constraints provided by the real world (not incidentally, these constraints are typically the scientific laws that they are to be discovering through the task!).

6) Teachers had difficulty changing their view of projects as capstones to projects as motivators for learning. Teachers would thus spend days of "preparation" before each part of a unit "helping students learn enough" before getting to the challenge. As a result, there was seldom enough time for iteration and to work through a complete design. The design challenge wasn't being used as a generator of questions, and the focus on science as inquiry was being lost as well. Furthermore, there was no rationale for class discussions, because the answers to the big issues had already been handed out with earlier readings or in lecture notes.

7) In some classes, design activities turned into arts and crafts.

Our formative assessment showed us that it takes the typical teacher two to three years to gain expertise in managing an inquiry classroom well. What could we do to help teachers manage an LBD classroom better right from the beginning? How could we make sure that teachers had adequate support and scaffolding as they learned to become effective LBD facilitators? In sum, we needed to find ways to help teachers help students (i) build a collaborative learning community (ii) value each others' ideas (iii) develop habits of thinking and communicating about science that relied on using data to justify their decisions and conclusions and (iv) focus on uncovering the science that underlies the constraints of their design challenge.

Although these issues did not at first glance seem like a cohesive set, many of these are the issues that are presented in the first chapter of most textbooks. It is in the first chapter that such questions as "what is science?" and section headings such as "the practice of science" appear. While it is also possible to introduce classroom values and science and design processes in the context of learning science content, our teachers warned us against that. They tried, and it was too difficult (Gertzman \& Kolodner, 1996; Hmelo et al, 2000). They asked us to give them a way of introducing those values and processes in a way that would allow them to refer back later as students were using the processes to learn content. Apollo 13 came from those discussions. It is designed both to scaffold teachers' development of an inquiry-based learning environment, and to introduce students to the rituals and practices of an LBD classroom.

\section{Goals of the Launcher Unit Introducing Design and Inquiry}

The unit has a series of short design challenges, each of which allows students to complete and present satisfactory solutions in a few class periods. These are followed by a single longer challenge (a week in length) that incorporates the skills introduced in the shorter challenges, helps the students and teacher learn to sustain the design cycle for a longer period, and embeds design and inquiry skills in the scientific discipline being studied.

\section{Introducing the Rituals of LBD}

One of the important ways to help both students and teachers maintain their focus on the goal of a design challenge is to incorporate methods for checking progress and impelling the project forward. In LBD, the class uses whiteboards (Barrows, 1985) to dissect a challenge, engages in pinup sessions (creating and presenting posters of 
ideas of how to attack the challenge), has messing about sessions (in which students work with materials to explore aspects of the challenge), conducts gallery walks (presentations of in-progress artifacts and hypotheses about their success in meeting the challenge), and so on. These rituals are introduced within the context of short design challenges, so that students understand their purpose and the expectations associated with each. As the students become comfortable with these rituals, they form a framework for the longer design cycles of the later units.

\section{Showing the Connection Between Science and Design}

To establish the connection between science and design, students need to see how building and testing their design is related to the scientific method. Middle school science classrooms usually serve as the introduction to how to frame hypotheses, how to test them, the importance of measurement, and the concepts of variables and control. Apollo 13's design challenges create the structure in which this vocabulary is introduced and in which these skills are first used. Design challenge statements include clear, measurable goals; additionally, they are set up so that comparisons between group solutions inevitably require the class to measure carefully. Moreover, students must agree on appropriate methods of measurement, of test control, and even of the variables to be measured. The habit of scientific method is established through the need to meet the design challenge.

As students work toward meeting a design challenge, they encounter obstacles that are part of the real world. For example, in building, the flexibility of the materials they are using may impede their ability to provide a solid platform; on the other hand, too-rigid materials may be unable to handle jarring or sheer forces. As students work to maximize the performance of their materials, they have to learn about their limitations. Through this, the teacher can introduce scientific concepts. The launcher unit provides several such experiences, so that students begin to look for the science underlying the constraints of design challenges they are trying to achieve.

\section{Creating a Collaborative and Scientific Culture}

The sum total of what students need to do in addressing the launcher unit's design challenges requires them to engage in practices of collaborative science and engineering. Better solutions come from building on ideas of others and from comparing and contrasting different design ideas to decide which has the most potential. Deeper understanding comes through sharing ideas with others and having others help explain or share their experiences. The only way to share ideas and build on what others have done is to have standards that are measurable and procedures that can be repeated. Teachers model and help students recognize these principles as they are enacted. Additionally, the unit uses movies and documentaries to model the attitudes we seek to engender.

\section{Providing Time for Teachers to Develop}

Teachers learn about LBD in a three-week summer workshop where they get experience with LBD both as students and as teachers (teaching students in a science summer program). However, in many ways the summer workshop is not an authentic teaching experience, and teachers find it more difficult to develop the inquiry-based culture in the world of daily classes and school district expectations. The launcher unit helps teachers focus on facilitating a small set of skills and rituals at a time.

\section{The Apollo 13 Unit}

The Apollo 13 unit is made up of a sequence of short activities, each lasting from one to seven class periods. The primary focus of each activity is introduction to processes that support the kinds of design and science activities students will engage in as they are learning science content during later units. The unit begins with viewing the movie Apollo 13, the 1995 movie about the aborted Apollo 13 mission to the moon. Each subsequent activity introduces students to aspects of the Learning by Design culture and gives them experience in the practices that will be important as they begin learning science content in an LBD setting.

In the film, students see scientists and engineers engaged in informed decision making, collaboration, inquiry, computation, clear communication, design, simulation, and so on. They hear scientific terms being used, and they see the complexities of devices and organizations and the need for clear terminology and collaboration skills. The movie provides memorable examples of scientific reasoning, design processes, and collaborative culture for the class to refer back to throughout their LBD work. An edited version of the movie takes one class period to show, and a second class period is devoted to discussing the model of science and design that the movie presents. After seeing the movie, we ask students to make their first attempt at a convincing argument - they need to use 
evidence from the movie to argue for or against continuation of the space program in a mock letter to their senator. Discussions about what makes for a persuasive argument introduce the need for deep knowledge and investigation.

Students then begin to engage in a series of design activities. The first, the Book Support Challenge, introduces them to collaboration, building on each other's ideas, design within constraints, design iteration, and the identification of design criteria. Students are divided into groups and given 10-15 minutes to build a bookstand out of 3" X 5" index cards, paper clips, and rubber bands. Their bookstand needs to support an open textbook well enough that pages can be turned. After 15 minutes (almost always enough time for all groups to be successful), the teacher leads the students through a simple gallery walk, in which each group describes their bookstand's design, shows how it works, answers questions, and solicits suggestions for improvement. The groups work a second time on the same challenge and show their revised designs in another gallery walk. The class is then given an additional challenge: they are to design the best-quality bookstand for the least amount of money (prices are fixed for each index card, paperclip, and rubber band). They follow the same procedure of designing and constructing in groups, sharing their designs and discussing them during gallery walks, and then iteratively revising.

During their second or third gallery walk, students invariably begin to accuse each other of copying. They've seen each other's ideas and identified some as better than their own or seen ways to integrate someone else's idea with one they already had. The accusations of copying provide the teacher with the opportunity to formally introduce students to the notions of collaboration and citation. Students learn that borrowing is essential for scientists and engineers as they move their fields forward. They are told that scientists and engineers use patents and reports to identify what they have learned or discovered, and other scientists and engineers cite those patents and reports as they find them useful to use. Students are encouraged to borrow ideas from each other and adapt them to fit their needs, so long as they cite the groups they are borrowing from.

As well, during this activity, the class engages in the activities and language of iterative design. They learn that the limitations of the materials they are using are what engineers call constraints and that the goals they are trying to achieve are what engineers and other designers call criteria. They learn that the revision cycle they are using is called iteration, and that one can almost never design a complex artifact correctly the first time - that iteration is the natural way among designers. They experience, as well, that when something doesn't work as well as they want, it's worth asking a question about why and finding out answers, and that designers do that too.

Next, we ask students to participate in a science investigation - one that requires control of the environment and procedure for replicability - the Oreo Cookie Challenge (1). We ask students to determine how many drops of icing will fit on a new kind of sandwich cookie. They encounter the importance of anticipating what variables need to be controlled, running pilot experiments, controlling variables, doing procedures consistently, and what it takes to be able to trust some other scientist's results. The class, as a whole, has to be able to make a recommendation to the cookie company about how many drops of icing their machines should be set to drop on each cookie half. Student groups each drop water on pennies to simulate dropping icing on cookies. When students first report their results to the class, they see that results are all over the place - some groups might have dropped 15 to 20 drops on their pennies, while other groups might have dropped up to 80 or even 100 . They are curious as to why. They examine each others' procedures, noticing that some hold the dropper higher than others, some drop water until it looks like the cookie will overflow on the next drop while others drop until water overflows and subtract one, some drop full drops while others drop small drops, some drop their water in the center, others on the side, and so on. They decide which are the most important variables and standardize those, and each group drops water on its pennies again using the new standardized procedure, reporting the results to the class. The second time, of course, results across groups are more consistent, but they still aren't consistent enough to make a recommendation. Students ask each other questions to discover other differences, standardize the procedure better, and try again, bringing more consistency. This activity seems to be an excellent one for helping students recognize the importance of controlling variables, the importance of doing procedures the same way every time, and the role of replicability in science investigation.

The unit continues with activities that focus on comparing and contrasting different designs ideas, choosing which ideas best address a challenge, using real-world cases to help understand a design challenge better, developing rules of thumb based on analysis, and carefully gathering data and keeping good records to facilitate justification of design changes. Each activity incorporates a subset of LBD's rituals and builds on the lessons learned from earlier activities. 
They finish the unit with a more complex design activity that requires substantial planning, that brings together all of the skills and rituals they've been learning over the previous weeks, and that begins to give them the opportunity to learn science in the context of design. The final activity in Apollo 13 lasts seven days. The final challenge for those students engaging in physical science is to design the slowest-falling parachute they can out of coffee filters and string. The parachute should hold two or three nickel-sized washers. The class begins by messing about with materials, taping filters together in different configurations to see how that effects the rate of fall. They continue by engaging in whiteboarding - identifying what they've noticed, ideas they have, and what they need to learn more about. Much of what they need to learn more about is how different properties of their parachutes will contribute to parachute performance. They pull these out as variables, and each group in the class chooses a different variable to test, holding the others constant. They report their results to the class, generating Parachute Rules of Thumb along the way. If other students don't believe the results of a group, they are encouraged to suggest ways of redesigning or running the experiment so that results will be more believable, and they send that group back for further investigations. Any groups that have generated believable results (not many the first time through) choose a different variable and investigate its effects on rate of fall. This is the first time they are generating rules of thumb that have specific science content - about air resistance, gravity, and their interactions, effects of mass, weight, and so on. We ask teachers to spend some time on these concepts so that students will recognize that they are learning science through design activities. However, we ask them to save longer discussion on these issues for a later unit on Newton's Laws (Kolodner et al, 1998).

After a believable set of rules of thumb has been created, each group designs its best. They share their ideas in a pin-up session. Every design decision must be justified based on the rules of thumb that have been generated through experimentation. When a group misunderstands a rule of thumb derived by another group, there is opportunity for discussion and further explanation and suggestions. Sometimes a group will ignore a rule of thumb, and others in the class point that out and make suggestions. After the pin-up session, each group revises its design ideas based on class discussion and moves on to construct their best parachute. A series of gallery walks are held as groups iterate toward their best designs. With each gallery walk, students have a chance to explain why some design decision they made wasn't a good one and why they changed it, and new rules of thumb might emerge. Once all groups are on their way to constructing successful parachutes, the students move towards a more competitive stance, iteratively moving toward their best without additional gallery walks. A contest is held at the end followed by comparison and discussion.

By the end of this activity, students have had significant experience designing experiments that test one variable at a time, controlling variables, testing a variable at several different levels, incorporating the results of several simple experiments into a design plan, and justifying design decisions based on many data sources. Throughout, doing is interspersed with reflection on the activities and what can be learned from them. Students have the opportunity to begin to learn skills in one challenge and then to apply them again in another challenge, and also discuss how their classroom activities, and the challenges they face, are similar to the experiences they've seen depicted in the film presentations and examples about real scientists and designers in their workbooks. Following the unit's activities, the class members summarize what they've experienced and learned, and anticipate when it might be useful.

\section{Discussion}

In most of our classes, by the end of Apollo 13, students are quite skilled at design talk (iteration, constraints, criteria), have learned to respect each other's ideas, and look forward to learning from each other. As well, they can talk about experimental procedure and recognize when variables have not been controlled or procedures not followed well. Launcher unit activities place their focus on developing the processes and culture of science and design, and introducing rituals that support these processes. They provide a shared set of anchors (CTGV, 1993) for later discussion and reference. Students continue to engage in each of the rituals and processes introduced in Apollo 13 over the course of the next LBD units. Following the launcher unit, students share a common language, rituals, and processes, so that the focus of subsequent challenges can be on science content and on refining their process skills. By introducing these skills early in an authentic context of use, early experiences can become anchors for further development of skills in additional and more varied contexts. 
It remains to be seen whether this is indeed the case. Our next steps include investigations necessary to ascertain the validity of our hypotheses: that focusing on the scientific and design skills and processes at the outset leads to their practice in project-based science learning environments, and that the science content is learned better as a result of preceding it with a focus on process. So far, we see that the launcher unit helps to instill the culture into the classroom that we think necessary to support inquiry-based learning of science. To understand the launcher unit's effects beyond that, we are looking for evidence of student remindings of what they did in this unit, their applications of what they are reminded of, and teacher use of experiences during the launcher unit to remind students of skills they might build on. We are also moving forward to focus the unit so that it takes only 3 weeks and to provide enough different variations on its activities so that some version of the unit could be used each year of middle school to set the stage for upcoming Learning by Design activities.

\section{Endnotes}

(1) We got this activity from Earl Carlyon, a teacher in Farmington, CT, who was inspired by an article in The Science Teacher, "Dealing with Data," by Art Christensen and Barbara Christian, Sept. 1997.

\section{References}

Barrows, H. S. (1985). How to design a problem-based curriculum for the preclinical years. NY: Springer.

Cognition and Technology Group at Vanderbilt. (1993). Anchored instruction and situated cognition revisited. Educational Technology, 33(3), 52-70.

Fasse, B. B. \& Kolodner, J. L. (2000). Evaluating classroom practices using qualitative research methods: Defining and refining the process. Proceedings of ICLS-2000 (this volume).

Gertzman, A. \& Kolodner, J. L. (1996). A Case Study of Problem-Based Learning in a Middle-School Science Class: Lessons Learned. Proceedings Second International Conference on the Learning Sciences, Evanston/Chicago, IL, July, 1996.

Hmelo, C.E., Holton, D.L. \& Kolodner, J.L. (2000). Designing to Learn about Complex Systems. Journal of the Learning Sciences.

Kolodner, J.L., Crismond, D., Gray J., Holbrook, J., \& Puntambekar, S.(1998). Learning by Design from Theory to Practice. Proceedings Third International Conference of the Learning Sciences '98, pp.16-22.

Puntambekar, S and Kolodner, J.L. (1998). The Design Diary: A Tool to Support Students in Learning Science by Design. Proceedings of ICLS 98. Atlanta, GA, 35-41.

\section{Acknowledgments}

This research has been supported by the National Science Foundation (ESI-9553583), the McDonnell Foundation, and the BellSouth Foundation. The views expressed are those of the authors. 